\title{
Characterization and evaluation of graphene oxide scaffold for periodontal wound healing of class II furcation defects in dog
}

This article was published in the following Dove Press journal: International Journal of Nanomedicine

\author{
Kohei Kawamoto' \\ Hirofumi Miyaji \\ Erika Nishida' \\ Saori Miyata' \\ Akihito Kato' \\ Akito Tateyama' \\ Tomokazu Furihata' \\ Kanako Shitomi' \\ Toshihiko Iwanaga ${ }^{2}$ \\ Tsutomu Sugaya \\ 'Department of Periodontology and \\ Endodontology, Faculty of Dental \\ Medicine, Hokkaido University, \\ Sapporo, Japan; ${ }^{2}$ Department of \\ Histology and Cytology, Faculty \\ of Medicine, Hokkaido University, \\ Sapporo, Japan
}

\begin{abstract}
Introduction: The 3-dimensional scaffold plays a key role in volume and quality of repair tissue in periodontal tissue engineering therapy. We fabricated a novel 3D collagen scaffold containing carbon-based 2-dimensional layered material, named graphene oxide (GO). The aim of this study was to characterize and assess GO scaffold for periodontal tissue healing of class II furcation defects in dog.
\end{abstract}

Materials and methods: GO scaffolds were prepared by coating the surface of a 3D collagen sponge scaffold with GO dispersion. Scaffolds were characterized using cytotoxicity and tissue reactivity tests. In addition, GO scaffold was implanted into dog class II furcation defects and periodontal healing was investigated at 4 weeks postsurgery.

Results: GO scaffold exhibited low cytotoxicity and enhanced cellular ingrowth behavior and rat bone forming ability. In addition, GO scaffold stimulated healing of dog class II furcation defects. Periodontal attachment formation, including alveolar bone, periodontal ligament-like tissue, and cementum-like tissue, was significantly increased by GO scaffold implantation, compared with untreated scaffold.

Conclusion: The results suggest that GO scaffold is biocompatible and possesses excellent bone and periodontal tissue formation ability. Therefore, GO scaffold would be beneficial for periodontal tissue engineering therapy.

Keywords: artificial collagen scaffold, cytotoxicity, nanocarbon, periodontal attachment, periodontal tissue engineering, rat cranial bone augmentation

\section{Introduction}

Periodontitis is a destructive inflammatory disease caused by oral bacterial cell growth that progressively invades periodontal tissue, such as alveolar bone, gingival connective tissue, periodontal ligament, and root cementum, subsequently diminishing the tooth support system. ${ }^{1}$ Currently, several periodontal tissue engineering therapies involving artificial bone grafting, ${ }^{2}$ guided tissue regeneration, ${ }^{3}$ and enamel matrix derivative ${ }^{4}$ clinically contribute to the repair of lost periodontal tissue. However, the effects of these strategies are insufficient in advanced periodontitis. Therefore, development of novel therapy and biomaterial is needed to improve the regeneration quantity and quality of periodontal tissue. It is well known that scaffold material is an important element in tissue engineering strategy. ${ }^{5,6}$ When a 3-dimensional (3D) scaffold is applied to a tissue defect, the scaffold provides a suitable environment for retaining growth and nutrition factors to facilitate the repopulation and differentiation of stem cells, ${ }^{7}$ blood vessels, and extracellular matrices, ${ }^{8,9}$ and consequent reconstruction of the lost tissue. ${ }^{10,11}$ Conventional periodontal regenerative therapy, like guided tissue regeneration and enamel matrix derivative, lacks
Correspondence: Hirofumi Miyaji Department of Periodontology and Endodontology, Faculty of Dental Medicine, Hokkaido University, NI 3 W7, Kita-ku, Sapporo 060-8586, Japan

Tel +8I II 7064266

Fax +8I II 7064334

Email miyaji@den.hokudai.ac.jp 
the application in tissue engineering scaffold. We speculate that advances in scaffold material to improve cellular responses and bioactive agent accumulation play a crucial role in periodontal regenerative therapy for advanced periodontitis.

Nanocarbon materials, constructed by 6-membered ring network structures, such as carbon nanotubes, ${ }^{12,13}$ carbon nanohorns, ${ }^{14}$ fullerene, ${ }^{15}$ and graphene, ${ }^{16,17}$ exhibit unique physical, chemical, and mechanical properties and are widely investigated for biomedical application. Another member of the nanocarbon family, graphene oxide (GO), is obtained by oxidation and exfoliation of graphite and comprises a carbon monolayer arranged in a 2-dimensional honeycomb lattice. ${ }^{18-20}$ GO exhibits a hydrophilic surface and has high dispersibility due to rich oxygen functional groups on its surface. ${ }^{21,22}$ Therefore, it is considered that GO surface physiologically absorbs active substances and agents via covalent bonding. Furthermore, GO exhibits cytocompatibility and promotes the proliferation and differentiation of stem cells. ${ }^{23}$

To apply GO to tissue engineering therapy, some investigators created GO composite scaffolds. Chen et al reported that nanocomposites, zinc oxide nanoparticles on carboxylated GO, induced bone tissue regeneration. ${ }^{24}$ Nair et al revealed that GO nanoflakes and gelatin-hydroxyapatite scaffolds promoted osteogenic differentiation of human mesenchymal stem cells. ${ }^{25}$ Furthermore, Xie et al fabricated GO/chitosan composite scaffold and its implantation facilitated rat skull bone augmentation in combination use with octacalcium phosphate, bone morphogenetic protein (BMP)-2, and Ag nanoparticles. ${ }^{26}$ Thus, GO composite scaffold would be beneficial for periodontal healing or regeneration via stem cell proliferation, bone induction, and consequent periodontal attachment reconstruction. Previously, we fabricated GOmodified collagen scaffold and assessed its biocompatibility. ${ }^{27}$ The scaffold exhibited high tissue compatibility in subcutaneous connective tissue and resulted in healing of tooth extraction sockets. ${ }^{28}$ Thus, we hypothesized that GO scaffold can stimulate the periodontal tissue-reforming process. However, GO scaffold application for periodontal defects has not been investigated thus far. Therefore, we herein report a new strategy for periodontal therapy using GO via a 3D collagen scaffold. We characterized and evaluated GO scaffold for periodontal wound healing of dog class II furcation defects.

\section{Materials and methods Fabrication of GO scaffold}

GO (nanoGRAX ${ }^{\circledR}$; Mitsubishi Gas Chemical Co. Inc., Tokyo, Japan) created by Hummers and Offeman method ${ }^{29}$ was dispersed into 1-methyl-2-pyrrolidone (Wako Pure Chemical Industries Ltd., Osaka, Japan; Figure 1A). The GO monolayer exhibited $<1 \mathrm{~nm}$ thickness and $20 \mu \mathrm{m}$ mean width. ${ }^{30}$ One hundred microliters of GO dispersion $(1 \mu \mathrm{g} / \mathrm{mL})$ was injected into the $3 \mathrm{D}$ collagen scaffold $(6 \times 6 \times 3 \mathrm{~mm}$, Terudermis $^{\circledR}$; Olympus Terumo Biomaterials Corp., Tokyo, Japan). After rinsing several times with ethanol and subsequent air drying, GO scaffold was obtained (Figure 1B). A collagen scaffold without GO was used as the control (Figure 1C).

\section{Assessment of morphology, compressive strength, and cytotoxicity of GO scaffold}

The morphology of GO scaffold was observed using a scanning electron microscope (SEM, S-4000; Hitachi Ltd., Tokyo, Japan) with accelerating voltage of $10 \mathrm{kV}$ after coating with a thin layer of Pt-Pd. The compressive stress of the scaffold at $50 \%$ compression was measured using a universal testing machine (EZ-S; Shimadzu Corp., Kyoto, Japan) with a crosshead loading speed of $0.5 \mathrm{~mm} / \mathrm{min}$.

Mouse osteoblastic MC3T3-E1 cells (RIKEN BioResource Center, Tsukuba, Japan) were seeded on the scaffold $\left(1 \times 10^{4}\right.$ cells/sample) and cultured in humidified $5 \% \mathrm{CO}_{2}$ atmosphere at $37^{\circ} \mathrm{C}$ in minimum essential medium (alpha-GlutaMAX ${ }^{\mathrm{TM}_{-}}$; Thermo Fisher Scientific Inc., Waltham, MA, USA) supplemented with $10 \%$ fetal bovine serum (Qualified; Thermo Fisher Scientific Inc.) and 1\% antibiotics (penicillin/streptomycin; Thermo Fisher Scientific Inc.). To assess the cytotoxicity, measurement of water-soluble tetrazolium salt (WST-8), and lactate dehydrogenase (LDH) level were carried out after 1, 3, and 5 days of culture using the Cell Counting Kit-8 (Dojindo Laboratories, Mashiki, Japan) and Cytotoxicity LDH Assay Kit-WST (Dojindo Laboratories), respectively. The absorbance of samples was read using a microplate reader (ETY-300; Toyo Sokki Co., Ltd., Yokohama, Japan) at 450 and $490 \mathrm{~nm}$. To assess the cell adhesion to the scaffold, samples seeded with MC3T3-E1 cells $\left(5 \times 10^{4}\right.$ cells/sample) for 1 day culture were washed with PBS and fixed with $3.5 \%$ formaldehyde. The samples were immersed in dissolving phalloidin $(1.5 \mu \mathrm{g}$, Actistain 555 Fluorescent Phalloidin, Cytoskeleton Inc., Denver, CO, USA) and 4',6-diamidino-2-phenylindole (2 $\mu \mathrm{g}$, Dojindo Laboratories) in $500 \mu \mathrm{L}$ of a bovine serum albumin solution (7.5\% w/v, Albumin Dulbecco's-PBS (-) Solution from bovine serum, Wako Pure Chemical Industries Ltd.) overnight at $4^{\circ} \mathrm{C}$. After washing 3 times with PBS, the stained cells were observed using a fluorescence laser scanning microscope (Biorevo BZ9000, Keyence Corp., Osaka, Japan).

\section{Evaluation of bone augmentation on rat cranial bone}

The in vivo experiments in rats were performed in accordance with the institutional animal use and care regulations 

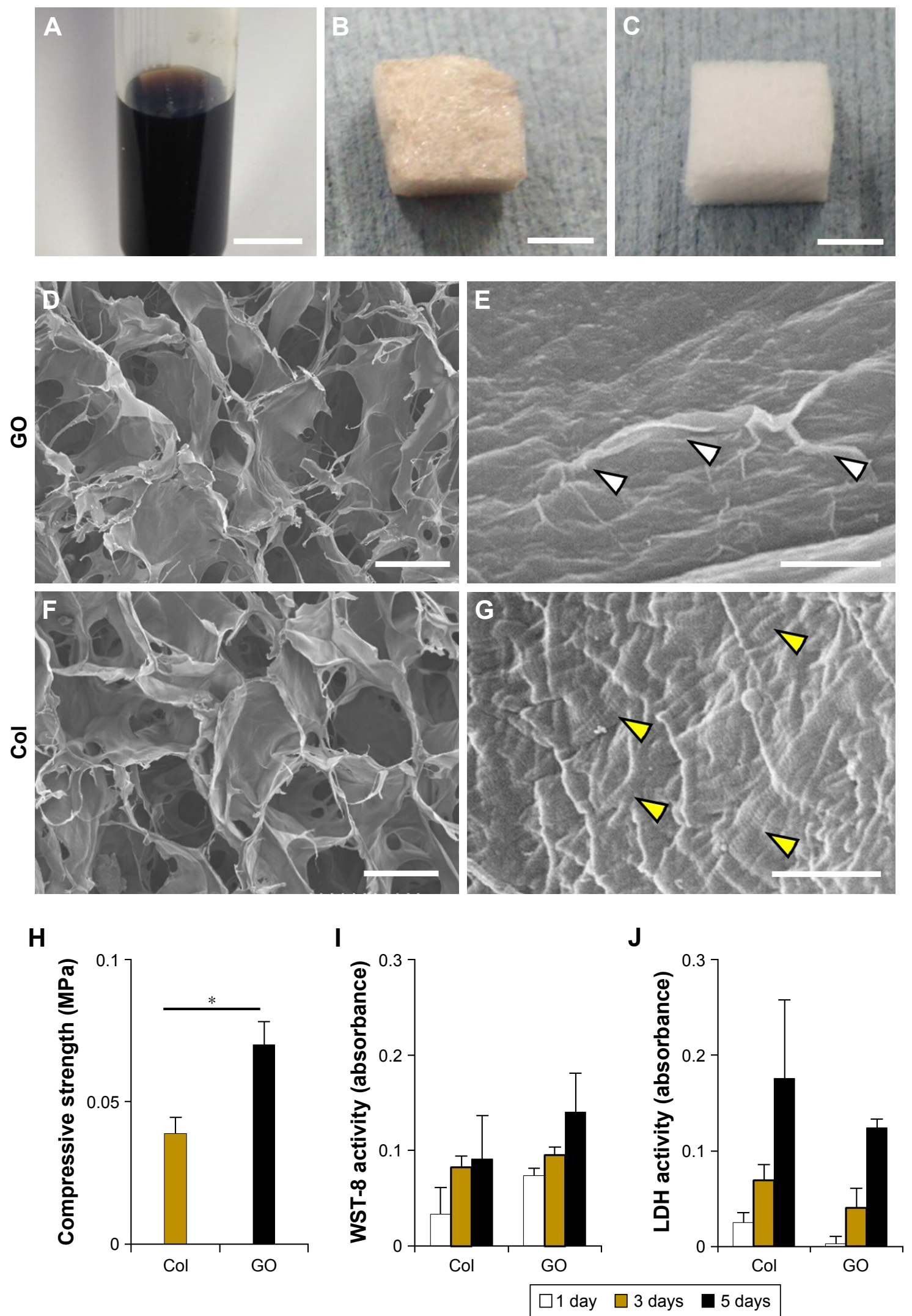

Figure I Characterization of GO scaffold.

Notes: (A) GO dispersing solution. (B) GO scaffold. (C) Collagen scaffold. (D, E) SEM images of GO scaffold. A wrinkled structure of GO (white arrowheads) was observed on collagen fibers of GO scaffold. (F, G) SEM images of collagen scaffold. Visible striations (yellow arrowheads) were shown on collagen fibers of untreated collagen scaffold. (H) Compressive strength of scaffolds ( $N=6$, mean $\pm S D$ ). $* P<0.05$. (I) WST-8 and (J) LDH activity of MC3T3E-I cells seeded onto scaffolds (N=4, mean \pm SD). Scale bars represent I cm (A), $3 \mathrm{~mm}(\mathbf{B}, \mathbf{C}), 100 \mu \mathrm{m}(\mathbf{D}, \mathbf{F})$, and I $\mu \mathrm{m}(\mathbf{E}, \mathbf{G})$.

Abbreviations: Col, collagen; GO, graphene oxide; LDH, lactate dehydrogenase; SEM, scanning electron microscopy, WST-8, water-soluble tetrazolium salt. 
of Hokkaido University (Animal Research Committee of Hokkaido University, Approval number 13-76) and approved by the Animal Research Committee of Hokkaido University. Thirty-four 10-week-old male Wistar rats weighing 190-210 g were given general anesthesia by intraperitoneal injection of $0.6 \mathrm{~mL} / \mathrm{kg}$ sodium pentobarbital (Somnopentyl; Kyoritsu Seiyaku Corp., Tokyo, Japan) and local injection of 2\% lidocaine hydrochloride with 1:80,000 epinephrine (Xylocaine Cartridge for Dental Use; Dentsply Sankin K.K., Tokyo, Japan). After exposing rat cranial bone, a $4 \mathrm{~mm}$ square area was decorticated using a dental bur. Subsequently, GO scaffold was placed onto the decorticated area and skin was sutured (BioFit-D 4-0; Washiesu Medical Co., Ltd., Tokyo, Japan). For control, implantation of untreated collagen scaffold or no implantation was performed. Then ointment containing tetracycline hydrochloride (Achromycin ointment; POLA Pharma Inc., Tokyo, Japan) was applied to the wound. At 10 and 35 days postsurgery, rats were euthanized using an overdose of sodium pentobarbital $(2 \mathrm{~mL} / \mathrm{kg})$.

Twelve samples, containing implanted GO scaffolds, cranial bone, and surround tissues, were collected at 10 days after implantation for histological observation. Samples were fixed in 10\% buffered formalin, decalcified in $10 \%$ ethylenediaminetetraacetic acid (EDTA) and embedded in paraffin. After thin slicing ( $6 \mu \mathrm{m}$ thick), sections were stained with H\&E and observed using light microscopy. To perform the histometric measurement, 3 stained sections were taken, 1 from the center of the excised tissue sample and 1 from tissue $1 \mathrm{~mm}$ to either side of the center. New bone height, new bone area, and residual scaffold area of 35-day specimens were measured using ImageJ software (ver. 1.41; National Institutes of Health, Bethesda, MD, USA).

Some samples were obtained for detection of macrophages, fibroblasts, and blood vessels. Anesthetized rats were perfused via the aorta with $4 \%$ formaldehyde. The tissues were pre-fixed in formaldehyde for $24 \mathrm{~h}$, dipped in $30 \%$ sucrose solution and embedded in optimal cutting temperature compound (Sakura Finetek Inc., Tokyo, Japan). After freezing in liquid nitrogen, $\sim 16 \mu \mathrm{m}$ thick sections were prepared and placed on poly-l-lysine-coated glass slides. After pretreatment with $0.3 \%$ Triton X-100 and normal donkey serum, sections were incubated overnight with the following primary antibodies: mouse anti-CD68 (1:100; Bio-Rad Laboratories Inc., Hercules, CA, USA), mouse anti-CD163 (1:100; Bio-Rad Laboratories Inc.), mouse antiprolyl-4-hydroxylase beta (P4HB; 1:1,600, clone 6-9H6; Acris Antibodies, Inc., San Diego, CA, USA), mouse anti- $\alpha$-smooth muscle actin (ASMA; 1:1,600, clone 1A4;
Sigma-Aldrich Co., St Louis, MO, USA) and mouse anti-rat endothelial cell antigen-1 (RECA-1; 1:1,000; AbD Serotec, Kidlington, UK). The antigen-antibody reaction sites were detected by incubation with Cy3-labeled anti-mouse IgG (Jackson ImmunoResearch Inc., West Grove, PA, USA). Nuclear staining was performed using TOTO-3 (Thermo Fisher Scientific Inc.). Sections were observed under a confocal laser scanning microscope (Fluoview; Olympus Corp.,). Sections incubated with normal mouse serum instead of respective primary antibody were used as negative controls.

Some samples were obtained for transmission electron microscopy (TEM) images. Using the standard procedure, samples were embedded in Epon. The samples were sliced and characterized using TEM (JEM1400, JEOL Ltd., Tokyo, Japan) at $80 \mathrm{kV}$ acceleration voltage.

\section{Assessment of periodontal healing of class II furcation defects}

Five healthy female beagle dogs, aged 12-16 months and weighing $\sim 10 \mathrm{~kg}$, were used for this experiment. The in vivo experiments in beagles were performed in accordance with the institutional animal use and care regulations of Hokkaido University (Animal Research Committee of Hokkaido University, Approval number 15-39) and approved by the Animal Research Committee of Hokkaido University. Surgical procedures were performed under general anesthesia with medetomidine hydrochloride $(0.4 \mathrm{~mL} / \mathrm{kg}$, Domitor; Nippon Zenyaku Kogyo Co., Ltd., Fukushima, Japan), butorphanol tartrate $(0.3 \mathrm{~mL} / \mathrm{kg}$, Vetorphale; Meiji Seika Pharma Co., Ltd., Tokyo, Japan) and midazolam $(0.3 \mathrm{~mL} / \mathrm{kg}$, Dormicum $^{\circledR}$ Injection $10 \mathrm{mg}$; Astellas Pharma Inc., Tokyo, Japan) under local anesthesia with lidocaine hydrochloride.

After reflection of the buccal flap, class II buccal furcation defects ( $5 \mathrm{~mm}$ in height, $3 \mathrm{~mm}$ in width) were surgically created at the maxillary second, third, and fourth premolars using a dental bur. The periodontal ligament and cementum were removed from exposed roots. Reference notches indicating the defect bottom were fabricated on root surfaces. Eighteen class II furcation defects were thus created and randomly assigned for scaffold implantation. Subsequently, root surfaces were irrigated using EDTA (Smear Clean; Nippon Shika Yakuhin Co., Ltd, Shimonoseki, Japan) and washed with saline. GO scaffold was applied to the defect and the flap was repositioned and securely sutured (Surgilon ${ }^{\mathrm{TM}}$; Tyco Healthcare Japan, Tokyo, Japan). As controls, untreated collagen scaffold was applied or no implantation was performed. After surgery, ampicillin sodium 
(300 mg/kg, Viccillin; Meiji Seika Pharma Co., Ltd.) was administered daily for 3 days and a plaque control regimen of $0.5 \%$ chlorhexidine (Hibitane ${ }^{\circledR}$; Sumitomo Dainippon Pharma, Tokyo, Japan) twice weekly was provided for the entire experimental period.

The animals were euthanized 4 weeks postsurgery using an overdose of sodium pentobarbital $(0.5 \mathrm{~mL} / \mathrm{kg})$ following general anesthesia with medetomidine hydrochloride $(0.1 \mathrm{~mL} / \mathrm{kg})$ and butorphanol tartrate $(0.1 \mathrm{~mL} / \mathrm{kg})$. The removed tissue blocks were fixed in $10 \%$ buffered formalin and analyzed by CT (AZ3000CT; Asahi Roentgen Ind. Co., Ltd, Kyoto, Japan). Subsequently, blocks were decalcified in $10 \%$ formic acid and embedded in paraffin wax. Sections (7- $\mu \mathrm{m}$ thick) along the mesio-distal plane were serially prepared, stained with H\&E and Masson's trichrome and observed using light microscopy. For histometric analysis, 3 stained sections were taken, 1 from the midsection of the furcation defect and 1 from section $1 \mathrm{~mm}$ to either side of the midsection. Five histomorphometric measurements, area of defect, newly formed bone, length of exposed root surface, cementum-like tissue, and periodontal ligament-like tissue, were evaluated for each stained section using ImageJ software (ver. 1.41; National Institutes of Health).

\section{Statistical analysis}

The mean and SD were calculated for each parameter. Differences between groups were analyzed using Scheffé's test. $P<0.05$ was regarded as statistically significant. All statistical procedures were conducted using SPSS 11.0 (IBM Corp., Armonk, NY, USA).

\section{Results \\ SEM observation, compression test, and cytotoxicity test of GO scaffold}

GO scaffold possessed interconnected inner-space architecture similar to untreated collagen scaffold. In higher magnification images, the scaffold surface was nano-modified by GO application. A thin layer of GO frequently formed wrinkles on the collagen fiber surface of the scaffold. Striations of collagen fibrils of scaffold fibers were not observed after GO modification (Figure 1D-G).

The compressive strength of GO scaffold and untreated scaffold are shown in Figure 1H. The compressive strength of GO scaffold was 1.7-fold greater than that of untreated scaffold (Figure 1H). Moreover, cytotoxicity assessments revealed that WST-8 and LDH levels in GO scaffold were equivalent to that of untreated collagen scaffold (Figure 1I and J).
In addition, fluorescence images showed that osteoblastic cell elongation and spreading were demonstrated on the GO scaffold, similar to untreated scaffold (Figure S1).

\section{Immunohistochemical observation of scaffold in rat}

Images at 10 days after implantation showed that CD68expressing macrophages and $\mathrm{P} 4 \mathrm{HB}$-expressing fibroblasts were increased in GO scaffold compared with untreated scaffold. In addition, CD163-expressing macrophages and a vascular-like structure formed by ASMA-positive smooth muscle cells and RECA-1-positive endothelial cells were detected in GO scaffold. By contrast, specimens of untreated scaffold rarely exhibited CD163-positive macrophages and the blood vessel-like structure (Figure 2).

\section{Rat cranial bone augmentation}

Images at 35 days after implantation showed that cranial bone induction continuous with existing bone was remarkably promoted by implantation of GO scaffold (Figure 3A-C). Slight residual GO scaffold was located around newly formed bone. Black spots were frequently dispersed into the bone marrow space and fibrous connective tissue around the newly formed bone. Ultrathin section of black spots showed the nanoscale flake-like structures resembling GO nanosheets in the cell body (Figure S2). Many cluster-like structures were found inside the cellular organelles like lysosome. Conversely, untreated collagen scaffold application showed no black spots and remarkable collagen residue on the cranial bone. The residue contained many fibroblastic cells, however, bone formation was slight (Figure 3D-F).

The results of histometric measurement are shown in Figure 3G-I. Newly formed bone area and height of samples receiving GO scaffold were 2.7- and 2.3-fold greater than those of untreated scaffold, respectively. In addition, residual scaffold area of untreated scaffold was 3.6-fold greater than that of GO scaffold.

\section{Periodontal healing of dog class II furcation defects}

Postoperative gingival healing was uneventful in all dogs (Figure 4A-C). Application of GO scaffold increased the degree of radiopacity of furcation bone defect in CT examination (Figure 4D-F). Histological observation revealed that $\mathrm{GO}$ application resulted in periodontal healing (Figure 5A-E). Newly formed alveolar bone was continuous with existing apical bone and mostly filled the furcation defect. The ankylotic union between root surface and alveolar 

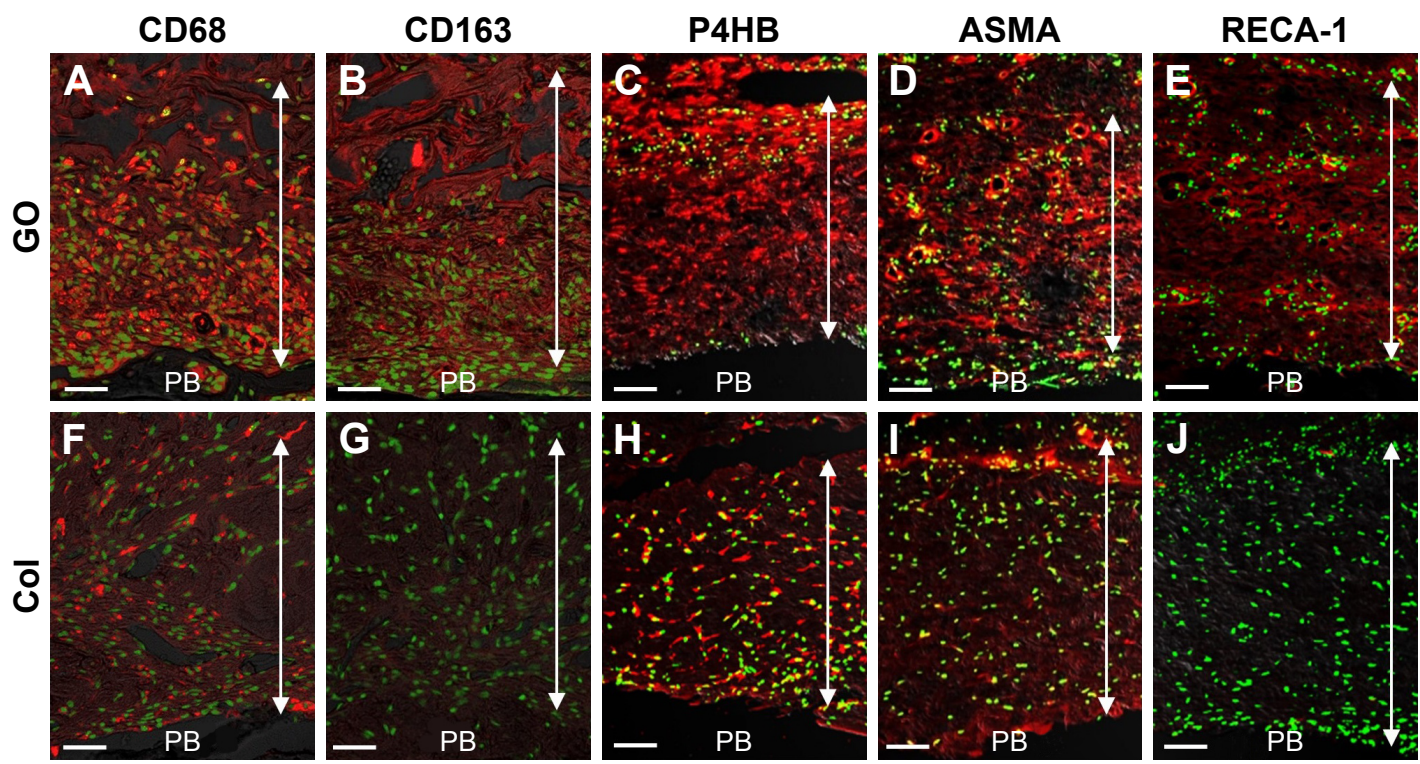

Figure 2 Immunohistochemical observation of GO scaffold.

Notes: (A, F) CD68, (B, G) CDI63, (C, H) P4HB, (D, I) ASMA, and (E, J) RECA-I, stained in red. Nuclei are stained green with TOTO-3. Double-headed arrows indicate the implanted scaffold. Scale bars represent $100 \mu \mathrm{m}$.

Abbreviations: ASMA, $\alpha$-smooth muscle actin; Col, collagen; GO, graphene oxide; P4HB, prolyl-4-hydroxylase beta; PB, preexisting bone; RECA-I, rat endothelial cell antigen-I.
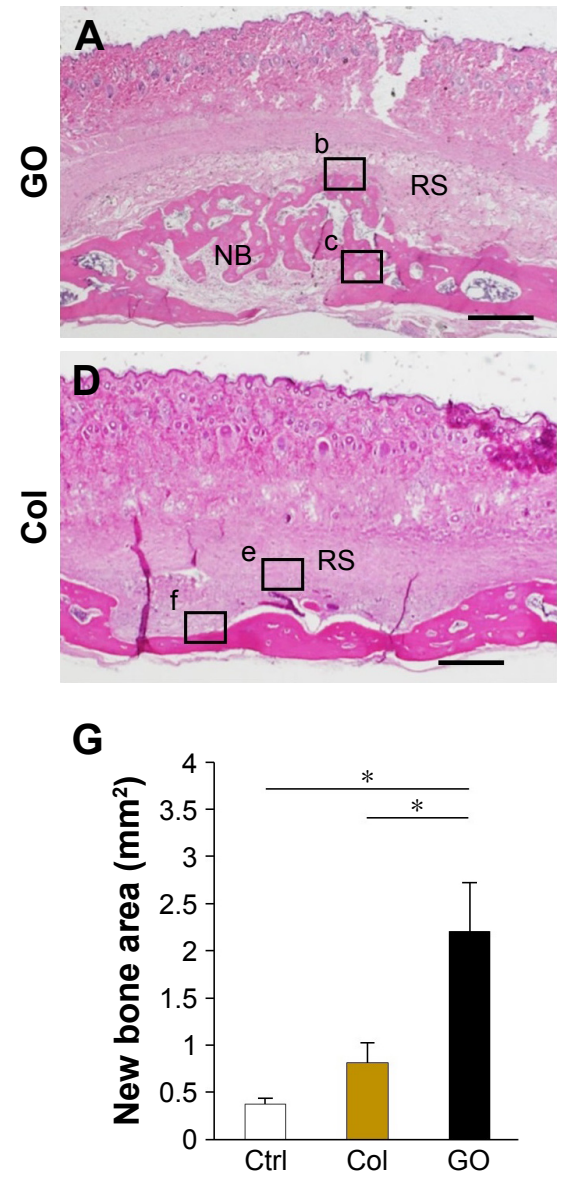
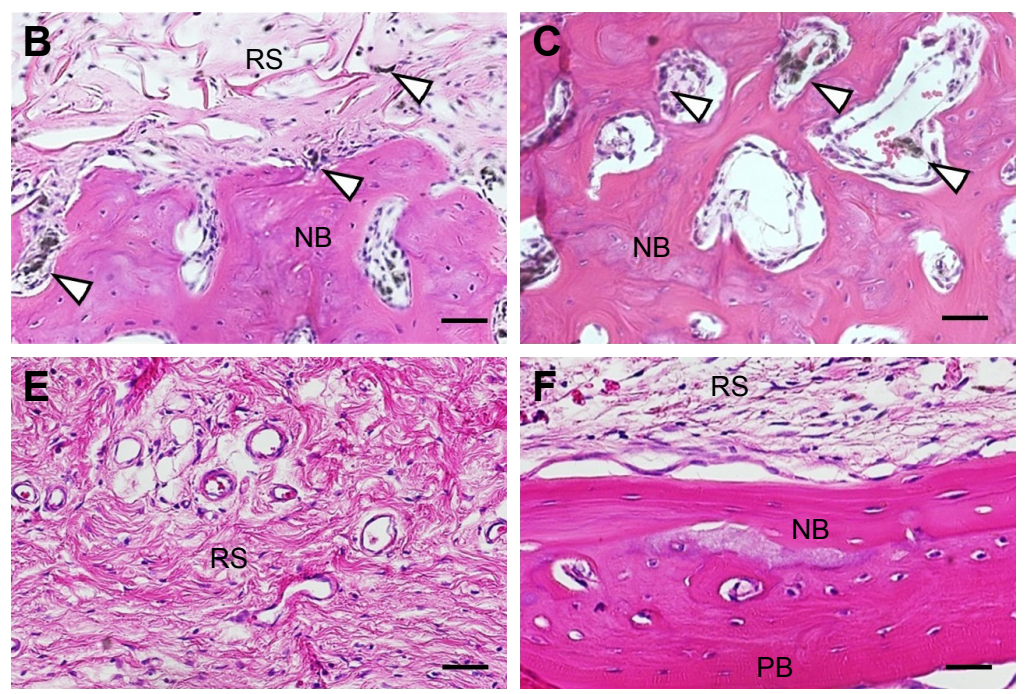

H
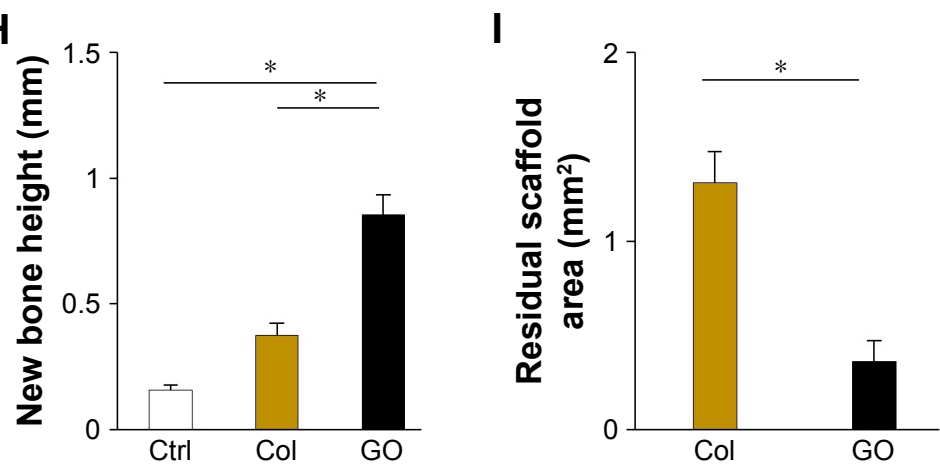

Figure 3 Histological findings after placement of GO scaffold on rat cranial bone.

Notes: (A-C) GO scaffold. High magnification (B, C) of the framed areas (b, c) in (A). Black spots (white arrowheads) were found around the newly formed bone. (D-F) Collagen scaffold. High magnification (E, F) of the framed areas (e, f) in (D). Staining with H\&E. Scale bars represent I mm (A, D) and I00 $\mu \mathrm{m}(\mathbf{B}, \mathbf{C}$, E, F). (G-I) Histomorphometric measurements $(\mathrm{N}=6$, mean $\pm \mathrm{SD}$ ) of new bone area $(\mathbf{G})$, new bone height $(\mathbf{H})$, and residual scaffold area $(\mathbf{I})$. $* \mathbf{P}<0.05$. Abbreviations: Col, collagen; Ctrl, control; GO, graphene oxide; NB, new bone; PB, preexisting bone; RS, residual scaffold. 

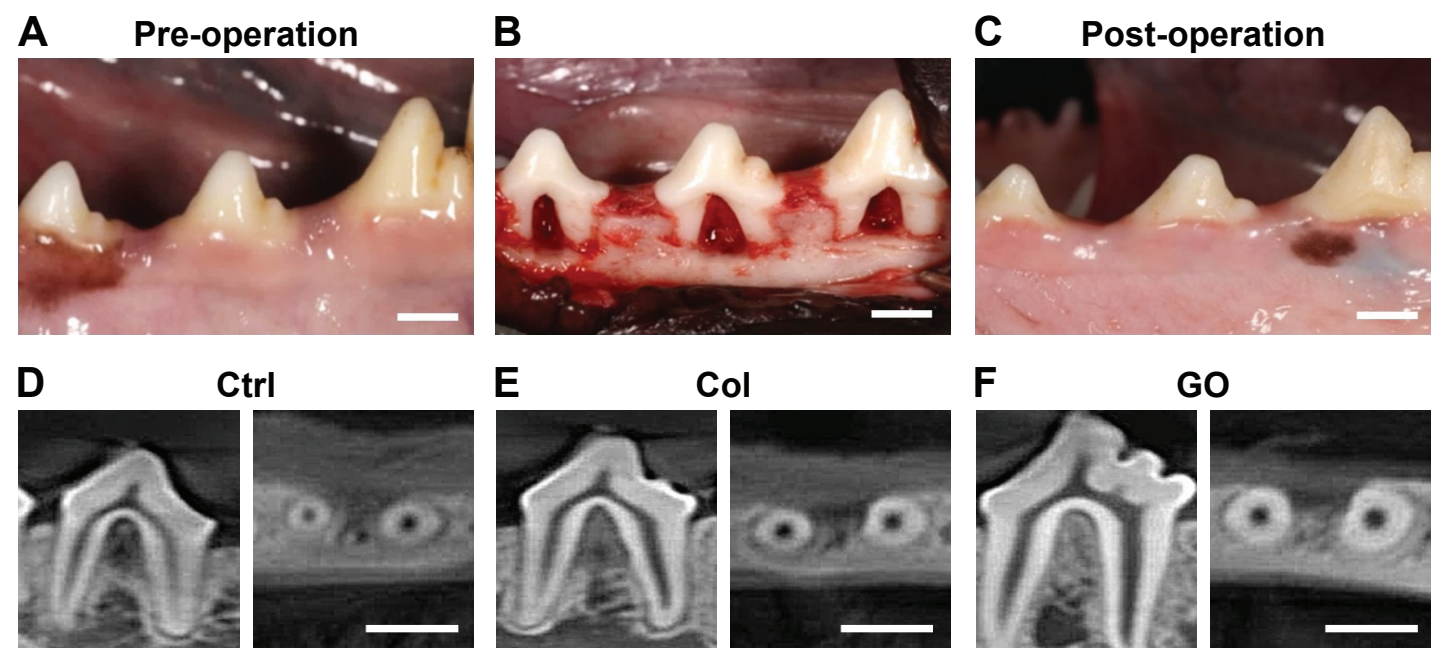

Col

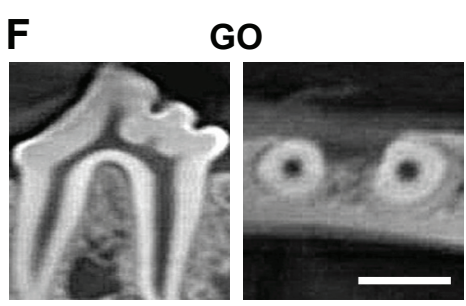

Figure 4 Digital photographs and CT images of dog class II furcation defects.

Notes: (A) Pre-operation. (B) Surgically created furcation defects. (C) Postoperation. (D-F) CT images of furcation receiving no implantation (D), collagen scaffold (E), and GO scaffold (F) at 4 weeks postsurgery. Scale bars represent $5 \mathrm{~mm}$.

Abbreviations: Col, collagen; Ctrl, control; GO, graphene oxide.

bone was not observed in all sections. In addition, severe root resorption was not demonstrated. Periodontal attachment apparatus was well formed in the furcation. Periodontal ligament-like tissue, cementum-like tissue, and alveolar bone with the insertion of Sharpey's fibers were observed on the root surface (Figure 5B and C). GO scaffold residue was rarely demonstrated in the furcation defect, and black spots were frequently found in the new bone marrow, similar to rat in vivo experiment (Figure 5D). In addition, black spots were found in the reformed periodontal ligament-like tissue (Figure 5E).

Untreated collagen scaffold implantation also promoted the formation of new bone and periodontal attachment in the furcation; however, the volume of regenerative tissue was less than that of GO scaffold (Figure 5F-H). Residual scaffold was frequently found in the central region of the furcation (Figure 5I). In the coronal portion, connective tissue occupied the furcation and few epithelial cells invaded into the furcation defect. Cementum-like tissue was rarely formed on the root surface (Figure 5J). In control specimens (no implantation group), bone and periodontal attachment was rarely repaired (Figure $5 \mathrm{~K}$ and $\mathrm{L}$ ). Inflammatory tissue, epithelial tissue, and plaque-like structure were found in the apical portion of the furcation (Figure $5 \mathrm{M}$ and $\mathrm{N}$ ).

Histometric analysis is shown in Figure 6. The mean alveolar bone area of the GO scaffold group (70\%) was significantly greater than those of untreated scaffold (36\%) and no implantation (14\%) groups (Figure 6A). In addition, periodontal ligament-like tissue and cementum-like tissue formation in the GO scaffold group were significantly higher than those in the other groups (Figure 6B and C).

\section{Discussion}

SEM observation revealed that GO covered the collagen fibers after injection of GO dispersion (Figure 1E). The GO-coated layer seemed to be very thin; however, GO application enhanced the strength of collagen scaffold (Figure 1H). Kanayama et al previously explained that the GO-coated layer creates an attractive force, van der Waals force, attributable to its nanoscale distance. ${ }^{31}$ Improvement of scaffold compressive strength may obtain space maintenance ability and promote regenerative tissue formation in the body. In addition, it was suggested that cytotoxicity of GO scaffold were approximately equivalent to that of untreated scaffold (Figure 1I and J). These results with regard to cytocompatibility support findings of previous studies. Ryoo et al reported that proliferation of NIH-3T3 fibroblasts was upregulated on the applied GO surface and concluded that the GO surface is biocompatible. ${ }^{32}$ Since the GO monolayer possesses many functional groups, including hydroxyl $(\mathrm{OH})$, epoxy $(\mathrm{C}-\mathrm{O}-\mathrm{C})$, and carboxyl $(\mathrm{COOH})$ species on its surface, the GO layer has a hydrophilic surface and contains electrostatic interactions. ${ }^{23,33}$ Reportedly, various bioactive substances, such as serum and extracellular matrix proteins, could be adsorbed on the GO surface to create a favorable environment for cell proliferation and differentiation. ${ }^{34,35}$ Indeed, Nishida et al reported that GO-applied scaffold was able to adsorb both albumin and lysozyme in the protein adsorption test. ${ }^{28}$ It is considered that adsorption properties of GO scaffold would be great advantages for scaffold material in tissue engineering.

In the rat implantation study, we confirmed that GO scaffold promoted bone augmentation of rat cranial bone. 

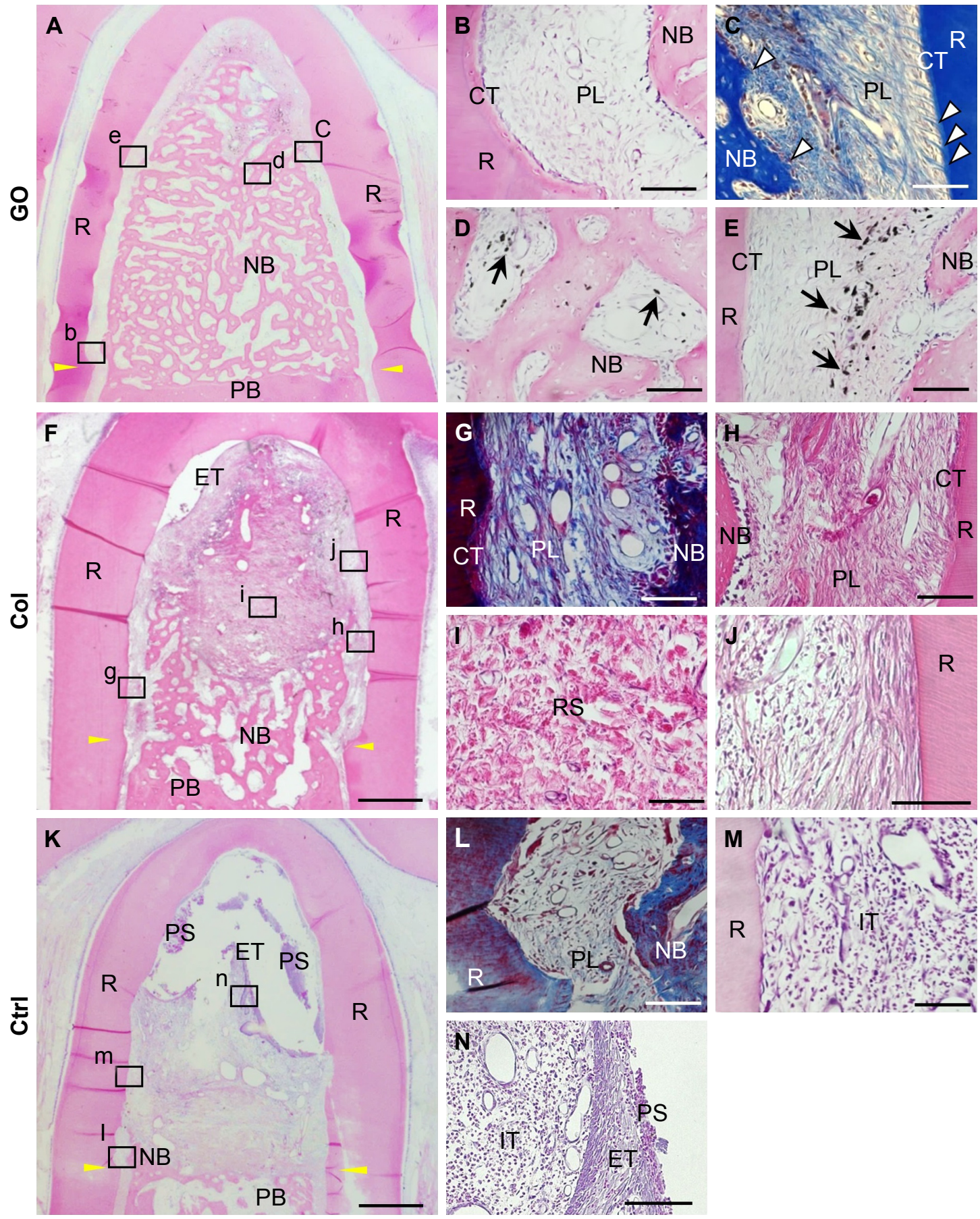

Figure 5 Histological findings after implantation of GO scaffold into dog class II furcation defects.

Notes: (A-E) GO scaffold. High magnification (B-E) of the framed areas (b-e) in (A). Periodontal ligament-like tissue, cementum-like tissue, and alveolar bone with the insertion of Sharpey's fibers (white arrowheads) was demonstrated. Black spots (black arrows) were found in the newly formed bone marrow and periodontal ligamentlike tissue. (F-J) Collagen scaffold. High magnification $(\mathbf{G}-\mathbf{J})$ of the framed areas $(\mathbf{g}-\mathbf{j})$ in $(\mathbf{F})$. (K-N) No implantation. High magnification $(\mathbf{L}-\mathbf{N})$ of the framed areas $(\mathbf{I}-\mathbf{n})$ in (K). Staining with H\&E (A, B, D-F, H-K, M, N) and Masson's trichrome (C, G, L). Scale bars represent I mm (A, F, K) and I00 $\mu \mathrm{m}(\mathbf{B}-\mathbf{E}, \mathbf{G}-\mathbf{J}, \mathbf{L}-\mathbf{N})$. Yellow arrowheads $(\mathbf{A}, \mathbf{F}, \mathbf{K})$ indicate the apical bottom of the periodontal defect.

Abbreviations: Col, collagen; CT, cementum-like tissue; Ctrl, control; ET, epithelial tissue; GO, graphene oxide; IT, inflammatory tissue; NB, new bone; PB, preexisting bone; PL, periodontal ligament-like tissue; PS, plaque-like structure; R, root; RS, residual scaffold.

Reportedly, GO application facilitates the proliferation and differentiation of osteoblasts. ${ }^{36,37}$ In the present study, immunostaining of 10 day cranial bone samples showed increased CD68-positive macrophages and P4HB-positive fibroblasts in GO scaffold compared with untreated scaffold. In addition, CD163-positive macrophages, ASMA-positive vascular smooth muscle cells, and RECA-1-positive endothelial cells associated with blood vessel formation were mainly found 

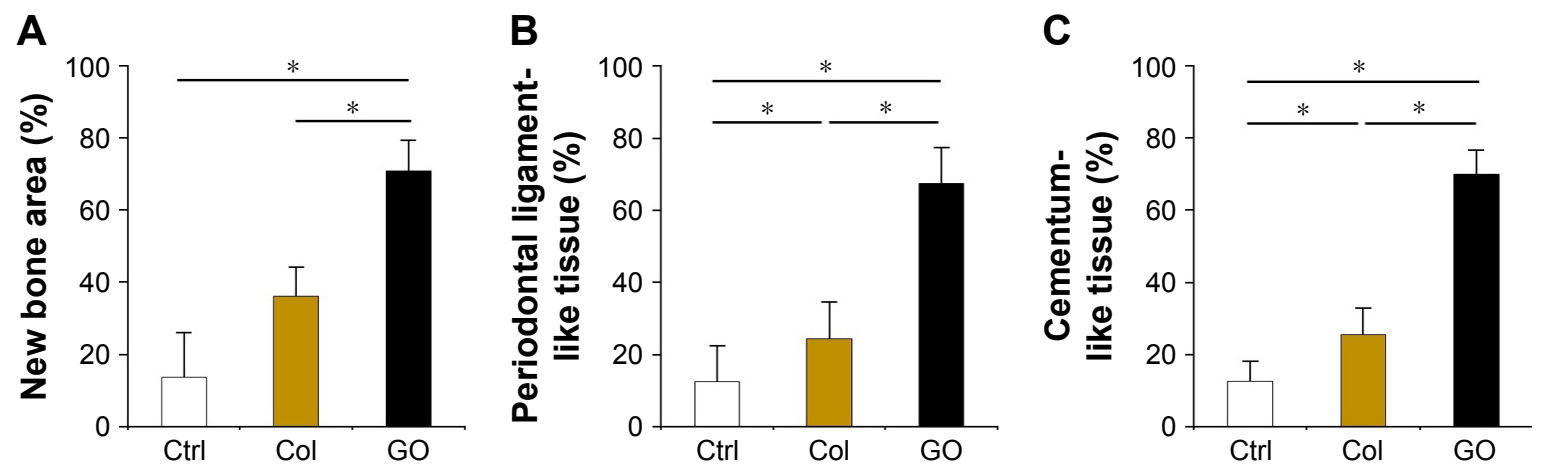

Figure 6 Histomorphometric assessment of dog class II furcation defects.

Notes: $(\mathbf{A}-\mathbf{C})$ Histomorphometric measurements $(\mathrm{N}=5$, mean $\pm \mathrm{SD})$ of new bone area $(\mathbf{A})$, periodontal ligament-like tissue $(\mathbf{B})$, and cementum-like tissue $(\mathbf{C})$. $* P<0.05$. Abbreviations: Col, collagen; Ctrl, control; GO, graphene oxide.

in GO scaffold (Figure 2). CD163-positive macrophages (M2 macrophages) are associated with immunosuppression and enhance tissue repair, remodeling, and subsequent wound healing. ${ }^{38}$ Polyl-4-hydroxylases have a major role in collagen biosynthesis and catalyze the formation of 4-hydroxyproline via the hydroxylation of prolines. Smooth muscle cells are located in the arterial wall to regulate blood delivery. According to our results, GO application may stimulate the cell migration and proliferation associated with wound healing process. Furthermore, Kanayama et al and Nishida et al reported that GO layer and GO scaffold exhibited calcium adsorption properties. ${ }^{28,31}$ Calcium-rich GO scaffold may induce osseous marker expression and adjust the in vivo environment for bone tissue engineering.

In the $\mathrm{CT}$ and histological images of postoperative dog class II furcation defects, GO scaffold promoted alveolar bone healing of class II furcation defects, similar to bone augmentation in the rat skull model. The regeneration of functional periodontal tissue generally requires periodontal attachment formation with simultaneous bone regeneration. In the present study, GO scaffold succeeded the formation of periodontal ligament-like and cementum-like tissue along with alveolar bone formation (Figure 5B and C). Proliferation of human periodontal stem cells has also been stimulated on GO layer, GO-coated titanium, and GO/fibroin film..$^{39,40}$ Therefore, the combination of $\mathrm{GO}$ and periodontal stem cells may be desirable for periodontal attachment formation. Miyaji et al previously reported that abnormal healing on the root surface, such as severe root resorption and ankylosis, were exhibited by implanting BMP, which strongly promoted osteoblastic differentiation and bone induction. ${ }^{41}$ In contrast to BMP therapy, aberrant healing was not found, and alveolar bone healing and restoration of the periodontal attachment was smoothly advanced by GO scaffold application. We thus confirmed the high potential of GO scaffold for periodontal tissue engineering.
The measurement of residual scaffold area (Figure 3I) revealed that GO application stimulated collagen scaffold degradation in the body. Wang et al revealed that GO was engulfed by cells, and residual graphene flakes were detected in cell lysosomes. ${ }^{42}$ In immunohistochemical observation, we detected many CD68-positive macrophages associated with phagocytosis in GO scaffold. In addition, black spots included GO flake-like structures and nanoscaled clusters (Figure S2), as well as in similar previous researches about GO internalization. ${ }^{42,43}$ Therefore, we considered GO was not decomposed but aggregated by macrophages through phagocytosis. GO scaffold completely disappeared in furcation defects in dog, and small black spots were found in newly formed periodontal tissues similar to the rat bone augmentation study (Figure 5D and E). Therefore, we speculate that GO application to collagen scaffold stimulated scaffold degradation and subsequently replaced the scaffold with regenerative periodontal tissue. The catalytic behavior of GO may play an important role in favorable tissue reconstruction by migration and proliferation of cells. Nonetheless, the influence of the aggregates should be addressed for biosafety. When GO was intravenously injected into rats, aberrant blood biochemical data and obvious organ damage were not observed, and GO was excreted with liver/spleen accumulates. ${ }^{44}$ However, the long-term effects of residual GO in periodontal and bone tissues are unclear. Further investigation is desirable for elucidation of GO scaffold biosafety.

\section{Conclusion}

We assessed the biological capabilities of 3D GO scaffold and effects of GO scaffold application on periodontal tissue healing in this preclinical study. Our results showed that GO application enhanced the cellular ingrowth behavior and angiogenesis on the rat skull. In addition, bone augmentation of rat cranial bone was promoted by GO scaffold implantation. 
Moreover, in the periodontal defect model of class II furcation in beagle dogs, alveolar bone healing and periodontal attachment repair were facilitated by GO scaffold application. Therefore, GO scaffold would be beneficial for periodontal tissue engineering therapy.

\section{Acknowledgments}

We are grateful to our colleagues, Natsumi Ushijima and Tsukasa Akasaka, for their assistance. We would also thank Mitsubishi Gas Chemical Co. Inc. for providing nanoGRAX ${ }^{\circledR}$, Olympus Terumo Biomaterials Corp. for providing collagen scaffolds and Nippon Shika Yakuhin Co., Ltd. for providing Smear Clean. This work was supported by JPSP KAKENHI Grant Numbers JP25463210, JP26870016, JP16K11822, and JP16H06604.

\section{Disclosure}

The authors report no conflicts of interest in this work.

\section{References}

1. Chapple IL, Genco R. Diabetes and periodontal diseases. Consensus report of the joint EFP/AAP workshop on periodontitis and systemic diseases. J Clin Periodontol. 2013;40(14):106-112.

2. Boyne PJ, James RA. Grafting of the maxillary sinus floor with autogenous marrow and bone. J Oral Surg. 1980;38(8):613-616.

3. Ehmke B, Rüdiger SG, Hommens A, Karch H, Flemmig TF. Guided tissue regeneration using a polylactic acid barrier. J Clin Periodontol. 2003;30(1):368-374.

4. Rathva VJ. Enamel matrix protein derivatives: role in periodontal regeneration. Clin Cosmet Investig Dent. 2011;3:79-92.

5. Yang S, Leong KF, Du Z, Chua CK. The design of scaffolds for use in tissue engineering. Part I. Traditional factors. Tissue Eng. 2001;7(6): 679-689.

6. Lutolf MP, Hubbell JA. Synthetic biomaterials as instructive extracellular microenvironments for morphogenesis in tissue engineering. Nat Biotechnol. 2005;23(1):47-55.

7. Li WJ, Tuli R, Huang X, Lauerriere P, Tuan S. Multilineage differentiation of human mesenchymal stem cells in a three-dimensional nanofibrous scaffold. Biomaterials. 2005;26(25):5158-5166.

8. Hutmacher DW. Scaffolds in tissue engineering bone and cartilage. Biomaterials. 2000;21(25):2529-2543.

9. Lutolf MP, Hubbell JA. Synthetic biomaterials as instructive extracellular microenvironments for morphogenesis in tissue engineering. Nat Biotechnol. 2005;23(1):47-55.

10. Chan BP, Leong KW. Scaffolding in tissue engineering: general approaches and tissue-specific considerations. Eur Spine J. 2008;17(4): 467-479.

11. Brien FJ. Biomaterials and scaffolds for tissue engineering. Mater Today. 2011;14(3):88-95.

12. Chico L, Crespi VH, Benedict LX, Louie SG, Cohen ML. Pure carbon nanoscale devices nanotube heterojunctions. Phys Rev Lett. 1996;76(6): 971-974.

13. Usui Y, Aoki K, Narita N, et al. Carbon nanotubes with high bone-tissue compatibility and bone-formation acceleration effects. Small. 2008; 4(2):20-26

14. Iijima S, Yudasaka M, Yamada R, et al. Nano aggregates of singlewalled graphitic carbon nano-horns. Chem Phys Lett. 1999;309(3-4): $165-170$.
15. Kroto HW, Heath JR, O’Brien SC, Curl RF, Smalley RE. C60: buckminsterfullerene. Nature. 1991;91(6):1213-1235.

16. Novoselov KS, Fal'ko VI, Colombo L, Gellert PR, Schwab MG, Kim K. A roadmap for graphene. Nature. 2012;490:192-200.

17. Chen GY, Pang DWP, Hwang SM, Tuan HY, Hu Y. A graphene-based platform for induced pluripotent stem cells culture and differentiation. Biomaterials. 2012;33(2):418-427.

18. Dikin DA, Stankovich S, Zimney EJ, et al. Preparation and characteriation of graphene oxide paper. Nature. 2007;448:457-460.

19. Dreyer DR, Park S, Bielawski CW, Ruoff RS. The chemistry of graphene oxide. Chem Soc Rev. 2010;39(1):228-240.

20. Marcano DC, Kosynkin DV, Berlin JM, et al. Improved synthesis of graphene oxide. ACS Nano. 2010;4(8):4806-4814.

21. Zhu Y, Murali S, Cai W, et al. Graphene and graphene oxide: synthesis, properties, and applications. Adv Mater. 2010;22(35):3906-3924.

22. Nanda SS, Papaefthymiou GC, Yi DK. Functionalization of graphene oxide and its biomedical applications. Crit Rev Solid State Mater Sci. 2015;40(5):291-315.

23. Lee WC, Lim CH, Shi H, et al. Origin of enhanced stem cell growth and differentiation on graphene and graphene oxide. ACS Nano. 2011; 5(9):7334-7341.

24. Chen J, Zhang X, Cai H, et al. Osteogenic activity and antibacterial effect of zinc oxide/carboxylated graphene oxide nanocomposites. Preparation and in vitro evaluation. Colloids Surf B. 2016;147(1):397-407.

25. Nair M, Nancy D, Krishnan AG, Anjusree GS, Vadukumpully S, Nair SV. Graphene oxide nanoflakes incorporated gelatin-hydroxyapatite scaffolds enhance osteogenic differentiation of human mesenchymal stem cells. Nanotechnology. 2015;26(16):161001.

26. Xie C, Lu X, Han L, et al. Biomimetic mineralized hierarchical graphene oxide/chitosan scaffolds with adsorbability for immobilization of nanoparticles for biomedical applications. ACS Appl Mater Interfaces. 2016;8(3):1707-1717.

27. Nishida E, Miyaji $\mathrm{H}$, Takita $\mathrm{H}$, et al. Graphene oxide coating facilitates the bioactivity of scaffold material for tissue engineering. Jpn J Appl Phys. 2014;53(6S):06JD04.

28. Nishida E, Miyaji H, Kato A, et al. Graphene oxide scaffold accelerates cellular proliferative response and alveolar bone healing of tooth extraction socket. Int J Nanomed. 2016;11:2265-2277.

29. Hummers WS Jr, Offeman RE. Preparation of graphitic oxide. $J$ Am Chem Soc. 1958;80(6):1339.

30. Hirata M, Gotou T, Horiuchi S, Fujiwara M, Ohba M. Thin-film particles of graphite oxide 1: high-yield synthesis and flexibility of the particles. Carbon. 2004;42(14):2929-2937.

31. Kanayama I, Miyaji H, Takita H, et al. Comparative study of bioactivity of collagen scaffolds coated with graphene oxide and reduced graphene oxide. Int J Nanomed. 2014;9:3363-3373.

32. Ryoo SR, Kim YK, Kim MH, Min DH. Behaviors of NIH-3T3 fibroblasts on graphene/carbon nanotubes. proliferation, focal adhesion, and gene transfection studies. ACS Nano. 2010;4(11):6587-6598.

33. Chen D, Feng H, Li J. Graphene oxide: preparation, functionalization, and electrochemical applications. Chem Rev. 2012;112(11):6027-6053.

34. Lee WC, Lim CH, Shi H, et al. Origin of enhanced stem cell growth and differentiation on graphene and graphene oxide. ACS Nano. 2011;5(9): 7334-7341.

35. Shi X, Chang H, Chen S, Lai C, Khademhosseini A, Wu H. Regulating cellular behavior on few-layer reduced graphene oxide films with wellcontrolled reduction states. Adv Funct Mater. 2012;22(4):751-759.

36. Liu H, Cheng J, Chen F, et al. Biomimetic and cell-mediated mineralization of hydroxyapatite by carrageenan functionalized graphene oxide. ACS Appl Mater Interfaces. 2014;6(5):3132-3140.

37. Zancanela DC, Simão AM, Francisco CG, et al. Graphene oxide and titanium: synergistic effects on the biomineralization ability of osteoblast cultures. J Mater Sci Mater Med. 2016;27:71.

38. Mantovani A, Biswas SK, Galdiero MR, Sica A, Locati M. Macrophage plasticity and polarization in tissue repair and remodeling. J Pathol. 2013;229(2):176-185. 
39. Zhou Q, Yang P, Li X, Liu H, Ge S. Bioactivity of periodontal ligament stem cells on sodium titanate coated with graphene oxide. Sci Rep. 2016;6:19343.

40. Vera-Sánchez M, Aznar-Cervantes S, Jover E, et al. Silk-fibroin and graphene oxide composites promote human periodontal ligament stem cell spontaneous differentiation into osteo/cementoblast-like cells. Stem Cells Dev. 2016;25:1742-1754.

41. Miyaji H, Sugaya T, Ibe K, Ishizuka R, Tokunaga K, Kawanami M. Root surface conditioning with bone morphogenetic protein-2 facilitates cementum-like tissue deposition in beagle dogs. J Periodont Res. 2010;45:658-663.
42. Wang K, Ruan J, Song H, et al. Biocompatibility of graphene oxide. Nanoscale Res Lett. 2011;6(1):8.

43. de Luna LA, de Moraes AC, Consonni SR, et al. Comparative in vitro toxicity of a graphene oxide-silver nanocomposite and the pristine counterparts toward macrophages. J Nanobiotechnology. 2016;14:12.

44. Yang K, Wan J, Zhang S, Zhang Y, Lee ST, Liu Z. In vivo pharmacokinetics, long-term biodistribution, and toxicology of PEGylated graphene in mice. ACS Nano. 2010;5(1):516-522. 


\section{Supplementary materials}
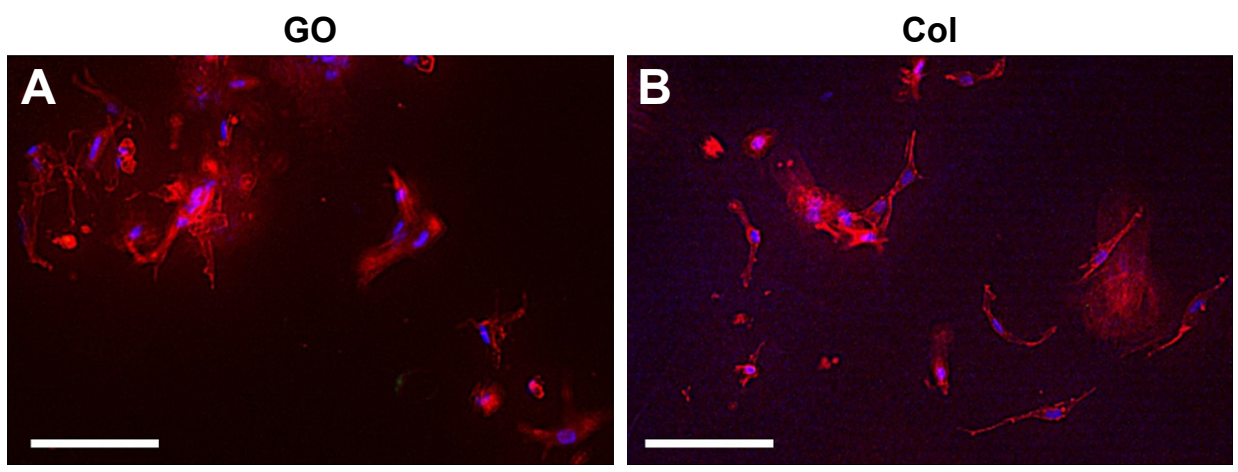

Figure SI Immunofluorescence images of cells cultured on GO scaffold.

Notes: (A) GO scaffold. (B) Collagen scaffold. Actin fibers are stained red with fluorescent phalloidin and nuclei are stained blue with DAPI. Scale bars represent I00 $\mu \mathrm{m}$. Abbreviations: Col, collagen; DAPI, 4',6-diamidino-2-phenylindole; GO, graphene oxide.
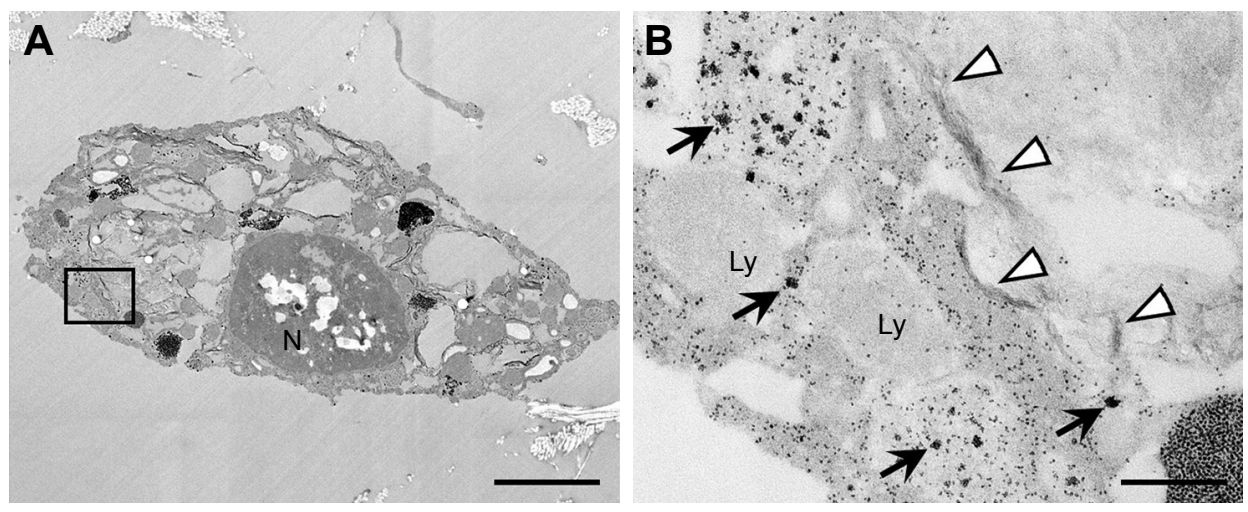

Figure S2 TEM images of cells after GO scaffold implantation.

Notes: (A) Blackened cell around the newly formed bone in rat. (B) Higher magnification of the boxed area in (A). Flake-like (arrowheads) and cluster-like structures (arrows) were observed in the cell body. Scale bars represent $2 \mu \mathrm{m}$ (A) and $200 \mathrm{~nm}$ (B).

Abbreviations: GO, graphene oxide; Ly, lysosome; N, nuclei; TEM, transmission electron microscope.

\section{Publish your work in this journal}

The International Journal of Nanomedicine is an international, peerreviewed journal focusing on the application of nanotechnology in diagnostics, therapeutics, and drug delivery systems throughout the biomedical field. This journal is indexed on PubMed Central, MedLine, CAS, SciSearch ${ }^{\circledR}$, Current Contents ${ }^{\circledR} /$ Clinical Medicine,
Journal Citation Reports/Science Edition, EMBase, Scopus and the Elsevier Bibliographic databases. The manuscript management system is completely online and includes a very quick and fair peer-review system, which is all easy to use. Visit http://www.dovepress.com/ testimonials.php to read real quotes from published authors. 